\title{
Loose bolt detection in a complex assembly using a vibro-acoustic sensor array
}

\author{
Philip Becht $^{\mathrm{a}, \mathrm{b}, *}$, Elke Deckers ${ }^{\mathrm{a}, \mathrm{b}}$, Claus Claeys ${ }^{\mathrm{a}, \mathrm{b}}$, Bert Pluymers $^{\mathrm{a}, \mathrm{b}}$, Wim Desmet ${ }^{\mathrm{a}, \mathrm{b}}$ \\ ${ }^{a}$ KU Leuven, Department of Mechanical Engineering, 3001 Leuven (Heverlee), Belgium \\ ${ }^{b}$ DMMS lab, Flanders Make, 3001 Leuven (Heverlee), Belgium
}

\begin{abstract}
A non-destructive testing strategy is proposed that combines microphone measurements and structural measurements in one array. The aim is to lower the total inspection time by reducing the number of structural sensors. This is possible by making use of the easier and faster installation of microphones as compared to structural sensors. Structural sensors are needed to limit the influence of background noise. The proposed algorithm is based on the theory of the Time-Reversal MUltiple SIgnal Classification (TR-MUSIC) algorithm. In addition to the novelty of using a vibro-acoustic array, this publication presents a strategy on how to select only the most 'valuable' regions of the frequency spectrum for the TR-MUSIC algorithm and an option to use the algorithm with a number of excitations below the theoretically necessary minimum.

The proposed procedure is applied in an experiment that targets the localisation of loose or removed bolts in an assembly of aluminium beams and a honeycomb panel, mounted with rivets, bolts and double-sided tape. Although none of the bolts is in the line of sight of the sensor array, all introduced defects can be found in this experiment. This includes the localisation of multiple defects as well. By adding artificial measurement noise to the microphone measurements, it is demonstrated that a combined vibro-acoustic array can cope with a high ambient noise level, while the process fails if a comparable measurement error is also applied to the structural sensors.
\end{abstract} Keywords: Non-destructive testing \& evaluation, Time-Reversal Multiple Signal

\footnotetext{
${ }^{*}$ Corresponding author

Email address: philip. becht@kuleuven. be (Wim Desmet)
}

Preprint submitted to Journal of Mechanical Systems and Signal Processing (ISSN: 0888-3270)May 14, 2019 


\section{Introduction}

The process of investigating the health status of a structure, which is out of operation without destroying it or adding additional damage is widely referred to as non-destructive testing and evaluation (NDT\&E) [1]. Many NDT\&E strategies are based on the mechanical vibrations of the test object, either introduced by ambient excitations, such as wind or waves or intentionally excited. Two of the most common and already industrially used methods are ultrasonic arrays and guided waves.

Ultrasonic arrays are arrays of transducers/sensors emitting an ultrasonic wave and recording its reflection. Primarily based on the time of flight of the reflected shortwavelength waves, it is possible to generate a highly detailed image of the area closely around the array. Also the inspection of larger surfaces is possible moving the array across the inspection surface. However, this process is time-consuming and therefore costly for large surfaces. Furthermore, the arrays contain typically a big number of transducers/sensors (the largest example known to the authors contains up to 256 independent channels [2]), which makes it difficult to couple all of them simultaneously to the surface of the test object, as e.g. the efforts made by Chatillon et al. [3] show. An additional restriction caused by the small inspection area nearby the array is, that ultrasonic arrays can only be applied for the investigation of accessible zones [4].

Guided wave methods excite specific wavemodes, which propagate through a test object. Also here, the location of defects can be determined measuring the time of flight of the reflected wavemodes. Though, for guided wave methods, care must be taken to measure the correct scattered wavemode, since the speed of propagation of different wavemodes varies due their dispersive behaviour. Following this procedure, a long range inspection is possible, setting aside the accessibility issues posed for the ultrasonic arrays. However, the application of these methods is restricted to simple test objects [5], since the discrimination of a reflection at a defect and a reflection at a geometrical feature must be possible [6]. Developers of these methods take a big effort to excite only the desired wavemode [7, 8] and as for ultrasonic arrays, also coupling 
liquids are needed for some transducer/sensor types [9].

As both strategies suffer from the effort that needs to be made to properly couple the test device to the test object, it seems logical to move to other types of sensors. Here microphones come to the fore [10], as the coupling to the test structure is automatically ensured by the surrounding air. In fact, the principle of listening to the sound radiated by a test object is used for centuries for quality control, e.g. checking a porcelain vase for cracks by tipping it and listening to the sound it makes. However, apart from the difficulty of combining this manual process with the high frequency guided waves or ultrasonic arrays (typically at frequencies far above the audible range), there are additional challenges since [11]:

- A manual process is an expensive procedure for mass produced products.

- The process is not reliable in an environment with severe background noise, which could be caused by workers speaking, welding, cutting or dropping things [12] or other machinery operating nearby the test object.

- No objective data can be documented and thus reproducing the test is difficult.

- A manual process relies on the worker's performance, which can lead to a "great variance in operation of NDT systems", partially due to ambient noise the worker is opposed to [12].

As the examples for noise sources imply, these harsh conditions for acoustic testing are present in nearly every production line. Thus the robustness requirements for all NDT\&E processes applied in a mass-production are set by these conditions. Against that background, in view of the ongoing evolution towards Industry 4.0, in order to overcome the shortcomings of manual testing and in order to fulfil normative requirements, automated testing becomes increasingly important [13].

Understanding NDT\&E as a 4 step process [14] with:

1 Detection: Is there damage in the test object?

2 Localisation: Where is the damage?

3 Assessment: How large is the damage? 
4 Consequence: How severe is the influence of the damage on the structural integrity?

this publication addresses step 2, trying to localise a damage in a complex assembly.

60

above, the microphones are coupled to the test object by the surrounding air, which automatically solves the coupling problem other types of sensors have to cope with.

Nevertheless, the new challenge of severe ambient noise arises by changing to microphone arrays. In order to overcome this, it is proposed to enhance the microphone need to be attached manually, but the expenditure of time decreases as compared to a fully structural array, since the number of structural sensors is smaller. In this context, a novel technique is presented dealing with the combination of different sensor types in one array for TR-MUSIC.

80

In order to improve the efficiency of the method in terms of total inspection time, the use of microphones is a significant improvement as compared to conventional sensors. The calculation time of the TR-MUSIC algorithm itself takes maximally some few seconds. A step in the test procedure that still provides room for a considerable speedup of the total inspection time is the excitation. Rather than coupled transducers, in the is explained how the number of necessary impact locations can be lowered, even below 
the theoretically given necessary minimum.

Furthermore, it is explained how to select only parts of the frequency spectrum to ensure a good localisation result even under non-perfect conditions.

The paper consists of a theoretical part followed by an experimental validation of the developed strategy. The test object consists of multiple aluminium beams and a honeycomb panel, mounted with bolts, rivets and double-sided tape and comes close to industrial complexity. The defects intended to be localised are loose or removed bolts at different connections, which are not visible for the sensor array.

\section{2. TR-MUSIC algorithm}

The aim of the first subsection in this section is to introduce the reader to the calculation of a damage index based on the TR-MUSIC algorithm. Based on this introduction to the algorithm, the following subsections explain:

- How to use the TR-MUSIC algorithm for undersampled data, meaning that the number of excitation locations and sensors is theoretically insufficient to generate an image of the inspected region.

- Why it is necessary to construct the damage index only based on the potentially valuable parts of the frequency spectrum. Furthermore, a proposal on how to select the 'potentially valuable parts of the frequency spectrum' is made.

- How to combine different sensor-types in one array.

\subsection{TR-MUSIC for damage localisation}

In the first step of a TR experiment, the signal transmitted by a source is measured by a sensor array. In a second step, the measured signal at each sensor is reversed in time and re-emitted at the sensors' locations. Due to the constructive interference of the re-emitted signals, temporal and spacial focusing on the primary source location is achieved [17, 18]. This process can be conveniently written in the frequency domain for an array of $N$ degrees of freedom (DOFs):

$$
\sum_{n=1}^{N} H_{n}(\omega) H_{n}(\omega)^{*} F(\omega)=a(\omega) F(\omega)
$$


with $H_{n}(\omega)$ being the transfer function from sensor element $n$ to the source location and $F(\omega)$ being the force input. The superscript $*$ denotes the complex conjugate of a function and $a(\omega)$ is a scalar, real value. From equation $(1)$, it can be seen that the spatial focusing happens independent of the frequency dependency of $a(\omega)$, since each term in equation (1) focuses at each frequency independently on the original source location. In order to obtain a correct reconstruction of the frequency content of $F(\omega)$, the coefficient $a(\omega)$ needs to be frequency-independent.

If the measured signal contains only the waves caused by the scattering of an incident wave-field at a scatterer, the scatterer can be regarded as the source. In consequence, the TR-process would focus on the scatterer's locations. In an experiment, the scattered field $\Psi_{s}\left(\omega, x_{n}, x_{m}\right)$ measured at $x_{n}$ due to an excitation at $x_{m}$ can be calculated by subtracting the known vibration of the intact structure $\Psi_{\text {init }}\left(\omega, x_{n}, x_{m}\right)$ from the actually measured vibration of the potentially defected structure $\Psi_{\text {meas }}\left(\omega, x_{n}, x_{m}\right)$ [19, 16]:

$$
\Psi_{s}\left(\omega, x_{n}, x_{m}\right)=\Psi_{\text {meas }}\left(\omega, x_{n}, x_{m}\right)-\Psi_{\text {init }}\left(\omega, x_{n}, x_{m}\right) .
$$

In order to facilitate readability, unless indicated differently, from equation (3) onwards, dependency on $\omega$ is assumed, but not specifically mentioned.

It can be shown [20, 16] that the scattered wave field can be re-written in the form

$$
\Psi_{s}\left(x_{n}, x_{m}\right)=P_{r}\left(x_{n}\right) \Theta P_{t} \rho\left(x_{m}\right),
$$

where $P_{t}$ and $P_{r}$ are the transmitter and receiver propagators. They describe respectively the propagation of a wave excited in domain $D_{t}$ to the entire inspection domain $D$ and the propagation from $D$ to the domain $D_{r}$, where the field is recorded. $P_{r}\left(x_{n}\right)$ indicates that only the propagation to the point $x_{n}$ is evaluated. $\rho\left(x_{m}\right)$ describes the excitation applied at $x_{m}$. The scattering itself is represented by $\Theta$.

Applying a singular value decomposition on $P_{r}$, it can be decomposed in its singular values $\lambda_{n}^{D}$ and singular functions $\Phi_{n}^{D}$ : 


$$
P_{r}=\sum_{n=1}^{\infty} \lambda_{n}^{D} \Phi_{n}^{D},
$$

where the superscript $D$ indicates the affiliation of the variables to the entire inspection domain.

An approximation of equation (4) is given in equation (5)

$$
P_{r} \simeq \sum_{n=1}^{N} \lambda_{n}^{D} \Phi_{n}^{D}
$$

where only the $N$ most relevant (largest) singular values and the corresponding singular vectors are kept.

In a similar manner, the transmitter propagator can be decomposed and truncated to the $M$ most relevant singular values:

$$
P_{t} \simeq \sum_{m=1}^{M} \lambda_{m}^{D} \Psi_{m}^{D} .
$$

The truncation factors $N$ and $M$ must be chosen such that the dynamics in the inspection domain can still be described entirely using only the singular functions $\Phi_{n}^{D}$ and $\Psi_{m}^{D}$. This condition implies that every scattering event at arbitrary scatterers of extent $D_{0}$ within the inspection domain $D\left(D_{0} \subset D\right)$ can be described by

$$
P_{r}^{D_{0}} \simeq \sum_{n=1}^{N} \lambda_{n}^{D_{0}} \Phi_{n}^{D_{0}}
$$

and

$$
P_{t}^{D_{0}} \simeq \sum_{m=1}^{M} \lambda_{m}^{D_{0}} \Psi_{m}^{D_{0}},
$$

where the superscript $D_{0}$ indicates the affiliation of the singular functions to the actually observed scattering event. It also follows that the space spanned by $\Phi_{n}^{D_{0}}$ and 
$\Psi_{m}^{D_{0}}$ is necessarily a subspace of (maximally the same dimension as) the space spanned by $\Phi_{n}^{D}$ and $\Psi_{m}^{D}$, respectively.

Introducing equations (7) and (8) into equation (3) leads to the expression for the scattered wave field

$$
\Psi_{s}\left(x_{n}, x_{m}\right)=\sum_{n=1}^{N} \sum_{m=1}^{M} \Phi_{n}^{D_{0}} \Lambda_{n, m}^{D_{0}} \Psi_{m}^{D_{0}} \rho\left(x_{m}\right) .
$$

The actual scattering event is summarised in $\Lambda_{n, m}^{D_{0}}$, which is composed of the singular values $\lambda_{n}^{D_{0}}$ and $\lambda_{m}^{D_{0}}$ and the scattering potential $\Theta$.

Assuming that the number of excitations $M$ and sensors $N$ is sufficiently large and well placed to illuminate the entire inspection region $D$, a matrix $K$ with elements $K_{n, m}=\Psi_{s}\left(x_{n}, x_{m}\right)$ can be constructed. It follows from equation (9) that the rank $R_{0}$ of this matrix is maximally $\min (M, N)$, but likely to be smaller. This results from the fact that the dimension of the space spanned by $\Phi_{n}^{D_{0}}$ and $\Psi_{m}^{D_{0}}$, respectively, is necessarily smaller or equal to the space spanned by $\Phi_{n}^{D}$ and $\Psi_{m}^{D}$, due to $D_{0} \subset D$. Therefore, the singular vectors $\boldsymbol{\Phi}_{n}^{D_{0}}$ and $\boldsymbol{\Psi}_{m}^{D_{0}}$ obtained from a singular value decomposition of $\boldsymbol{K}$ can be grouped into two orthogonal spaces. On the one hand side the signal space, $S_{\Psi}$ and $S_{\Phi}$, respectively, which is spanned by the singular functions describing the physical wave propagation from the excitation array to the scatterer(s) and from the scatterer(s) to the sensor array, respectively. Those singular vectors correspond to the $R_{0}$ largest singular values. On the other hand side the noise space, $N_{\Psi}$ and $N_{\Phi}$, which has no physical interpretation, but the mathematical property of being orthogonal to the signal space:

$$
\begin{aligned}
& S_{\Phi} \oplus N_{\Phi}, \\
& S_{\Psi} \oplus N_{\Psi} .
\end{aligned}
$$

If a vector $\boldsymbol{g}(z)$ with each element containing the transfer function from a random point $z$ in the inspection domain to one sensor in the sensor array lies completely in $S_{\Phi}$, then it follows from the properties in (10) for the multiplication with a noise space 175 singular vector $\boldsymbol{\Phi}_{n} \in N_{\Phi}$ : 


$$
\boldsymbol{g}(z)^{\mathrm{H}} \boldsymbol{\Phi}_{n}=0, \text { for } R_{0}<n \leq N .
$$

If $\boldsymbol{g}(z)$ also contains parts of $N_{\Phi}$, then:

$$
\boldsymbol{g}(z)^{\mathrm{H}} \boldsymbol{\Phi}_{n} \neq 0, \text { for } R_{0}<n \leq N,
$$

From the discussion of the TR-principle, it is known that the best possible focus on the source location is reached by back-propagating the time-reversed version of the measured signal. Since all the information on the physical propagation from the sensors to the defect(s) is contained in $S_{\Phi}$, it follows that if equation 111 is fulfilled, $z$ is a point in the defect domain and the vector $\boldsymbol{g}(z)$ can be interpreted as the best possible focus on this part of the scatterer(s) that can be achieved with a given array.

Exploiting the properties of equation (11) and (12), the TR-MUSIC algorithm is defined as [19, 21]:

$$
I(z)=\left(\left|\boldsymbol{g}(z)^{\mathrm{H}} \mathbf{\Phi}_{n}\right|\right)^{-1}, \text { for } R_{0}<n \leq N
$$
a scatterer can be replaced by finding the location $z$ linked to the vector $\boldsymbol{g}(z)$ for which equation (13) reaches its maximum. Equation (13) holds for each vector $\boldsymbol{\Phi}_{n} \in N_{\Phi}$ individually. In order to exploit the orthogonality of all $\boldsymbol{\Phi}_{n}$ 's to $N_{\Phi}$, it is common practice to sum equation (13) over the entire noise space, increasing the dynamic range and the robustness of the pseudospectrum $I(z)[19,22,23,24,25,26,27,28,29]$ :

$$
I(z)=\left(\sum_{n=R_{o}+1}^{N}\left|\boldsymbol{g}(z)^{\mathrm{H}} \boldsymbol{\Phi}_{n}\right|\right)^{-1} .
$$

Since the location of the maxima of $I(z)$ are linked to the location of the scatterers, they appear at the same spot, independent of the frequency at which equation (14) is evaluated. Again in order to increase robustness and dynamic range of the localisation 
algorithm, an additional summation over different frequencies was successfully applied, e.g. by Yavuz and Teixera [24] (and before using the signal space singular vectors [30]), Marengo et al. [20] and Becht et al. [16].

Following the same reasoning, equation (14) can also be derived for the imaging from excitation side using $\boldsymbol{\Psi}_{n}$ with $R_{0}<n \leq M$.

In all examples presented in this publication, the vector $g(z)$ is normalised using the routine described in Appendix B in order to limit the influence of modal behaviour on the detection process.

It should be pointed out that alternative strategies to compute $I(z)$ and to compose $\boldsymbol{K}[31,32]$ have been published in literature. Especially the computation of the defectindicating function $I(z)$ offers opportunities to adapt the algorithm according to specific needs. On the one hand, the signal space could be used instead of the noise space, which gives a physical meaning to the focusing amplitude and, if performed fully experimentally in time-domain, this approach automatically compensates for variances in the propagation medium. On the other hand, the resolution of the MUSIC-based pseudospectrum is superior and the computational time of the algorithm in equation (14) significantly smaller than for a full time-domain simulation. In the comparison of the robustness against random noise, both computational signal and noise space methods perform similarly [22, 33]. Furthermore, Some authors use a square like expression $\left(\boldsymbol{g}(z)^{\mathrm{H}} \boldsymbol{\Phi}_{n} \boldsymbol{\Phi}_{n}^{\mathrm{H}} \boldsymbol{g}(z)\right)$ instead of $\boldsymbol{g}(z)^{\mathrm{H}} \boldsymbol{\Phi}_{n}$ in equation 14, , which automatically makes the elements in the sum in equation (14) real and positive and hence replaces the operation $|\bullet|[31]$. However, in both cases the intrinsic idea is the exploitation of the orthogonality between signal and noise space, which holds independent of the use of a linear or a squared term.

\subsection{TR-MUSIC with undersampled data}

For a large number of defects or a volume-defect that scatters the incident wave-field in a very complex manner, the number of necessary sensors $N$ and excitations $M$ to completely capture the scattering event is likely to become very large. Therefore, in the majority of the application cases found in literature, large arrays (e.g. 48 and 96 DOF transmitter/sensor array [27]) are employed. If, due to practical considerations either $M$ 
or $N$ are restricted and smaller than $R_{0}$, the rank of $\boldsymbol{K}$ becomes $R_{o}^{\text {meas }}=\min (M, N)<$ $R_{0}$. In consequence the number of signal space singular vectors is not sufficient to capture all possible scattering events in the observed domain. This means that the space spanned by the found signal space singular vectors $S_{\Psi}^{\text {meas }}$ or $S_{\Phi}^{\text {meas }}$, respectively, is only a part of the theoretical signal space $S_{\Psi}$ or $S_{\Phi}$. Therefore, the space spanned by the found noise space singular vectors $N_{\Psi}^{\text {meas }}$ or $N_{\Phi}^{\text {meas }}$ is no longer guaranteed to be orthogonal to $S_{\Psi}$ or $S_{\Phi}$, respectively, which causes the TR-MUSIC algorithm as described in equation (14) to break down.

An example for this case can be found in figure 1 . where a $1.5 \mathrm{~mm}$ thick steel plate $(1.608 \mathrm{~m} \times 1.848 \mathrm{~m})$ is excited by $M=11$ point forces normal to the surface. In order to facilitate the illustration, the same locations are used to measure the out-of plane 235 acceleration $(N=11)$. In total there are $R_{0}=6$ point masses, which act as point scatterers in the observation domain (light green). The image constructed based on the 5 noise space singular vectors (for point scatterers under the Born approximation it is known that the dimension of the signal space is equal to the number of scatterers [34, 35, 19]) at $1240 \mathrm{~Hz}(\lambda=12 \mathrm{~cm})$ is presented in figure $2 \mathrm{a}$. As can be seen, the locations of all 6 point scatterers are highlighted, although the one in the bottom centre is difficult to identify. Although there is a high dynamic range between detected scatterers and background noise, in the background of the image still artefacts of the dominant modeshape at this frequency are visible.

Since the scatterers' position is independent of the frequency, the location of the pixels with the largest amplitude will not change as a function of frequency and always indicate the scatterers. Equation 111 holds and thus $I(z)$ tends towards infinity (in reality a very high, but finite value). At the same time, the pseudospectrum's amplitude at pixels $z$ not correlating with a scattering event does depend on the test function, since the finite value in equation (12) and in consequence also $I(z)$ changes with frequency.

Making use of this knowledge, even under these ideal conditions (no noise, perfect model), the dynamic range of the image can be improved by introducing a multiplication over different frequencies in equation (14): 


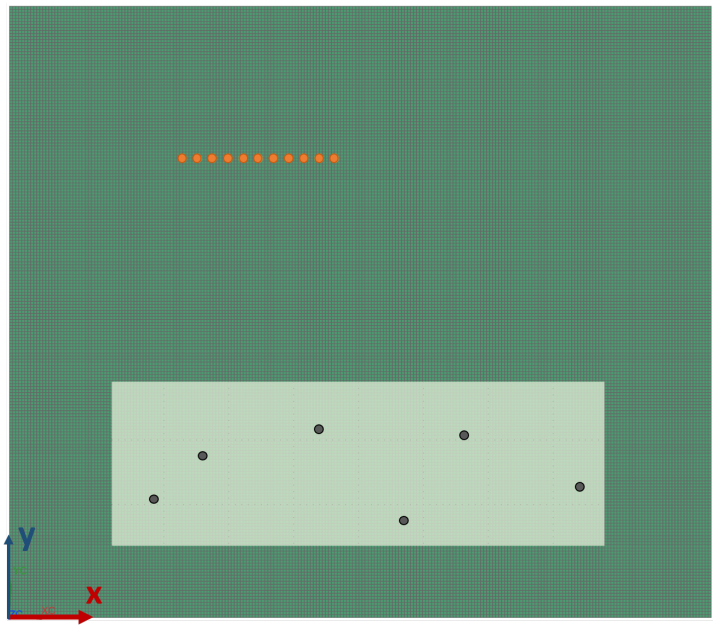

Figure 1: FE model of a $1.608 \mathrm{~m} \times 1.848 \mathrm{~m}$ plate with 11 excitation/sensor locations (orange), 6 point scatterers (black) and the observation domain indicated in light green.

$$
I(z)=\left(\prod_{f=1}^{N_{f}} \sum_{n=R_{0}^{\text {meas }}+1}^{N}\left|\boldsymbol{g}_{f}(z)^{\mathrm{H}} \boldsymbol{\Phi}_{n, f}^{\text {meas }}\right|\right)^{-1},
$$

with $N_{f}$ being the number of frequency evaluations.

This technique was suggested by Solimene et al. [28, 36] and is referred to as 255 interferometric TR-MUSIC. The effect on the image can be seen in figure 2 .

The case described in the beginning of this section, where $M=4=R_{0}^{\text {meas }}<R_{0}=6$ evaluated at a single frequency (equation (14) can be found in figure 2 . As expected, the algorithm is not able to correctly split $S_{\Phi}$ from $N_{\Phi}$, which is why the detection fails.

However, the measured signal space is still a subset of the true signal space, $S_{\Phi}^{\text {meas }} \subset S_{\Phi}$. Since in this example, the space spanned by the $N=11$ sensors did not change as compared to the ideal scenario, it follows that the true noise space is a subset of the estimated noise space, $N_{\Phi} \subset N_{\Phi}^{\text {meas }}$. As a consequence, the measured signal space is still orthogonal to the true noise space, $S_{\Phi}^{\text {meas }} \oplus N_{\Phi}$. This condition contributes correct information to the pseudospectrum computed according to equation 265 14. In addition to this, $I(z)$ is polluted by the contribution of the vectors in $N_{\Phi}^{\text {meas }}$, which are not part of $N_{\Phi}$. This is the reason for the image in figure 2 to fail. 


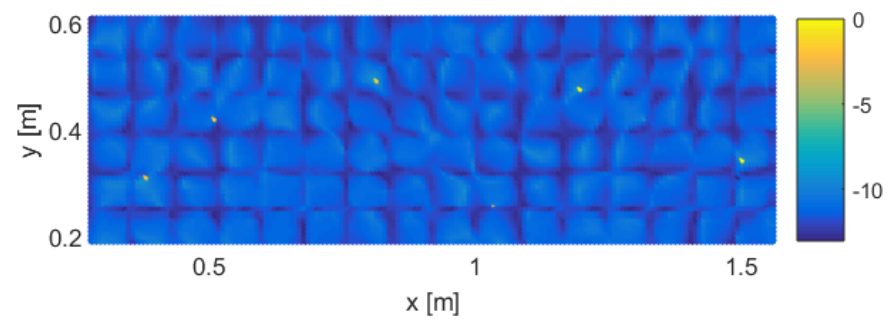

(a) Damage index based on $M=N=11, R_{0}=R_{0}^{\text {meas }}=6$ at $1240 \mathrm{~Hz}$. Logarithmic color scale.

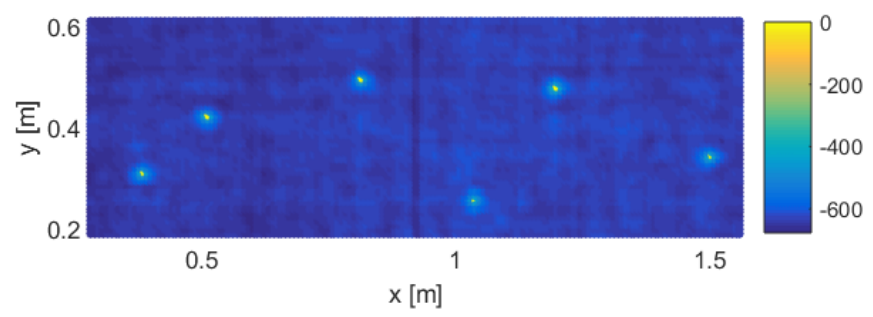

(b) Damage index based on $M=N=11, R_{0}=R_{0}^{\text {meas }}=6$ for 151 frequency lines between $500 \mathrm{~Hz}$ and $1000 \mathrm{~Hz}$. Logarithmic color scale.

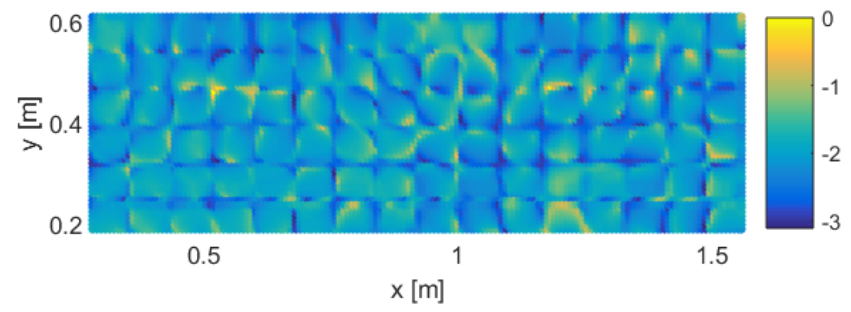

(c) Damage index based on $M=4=R_{0}^{\text {meas }}<R_{0}=6, N=11$ at $1240 \mathrm{~Hz}$. Logarithmic color scale.

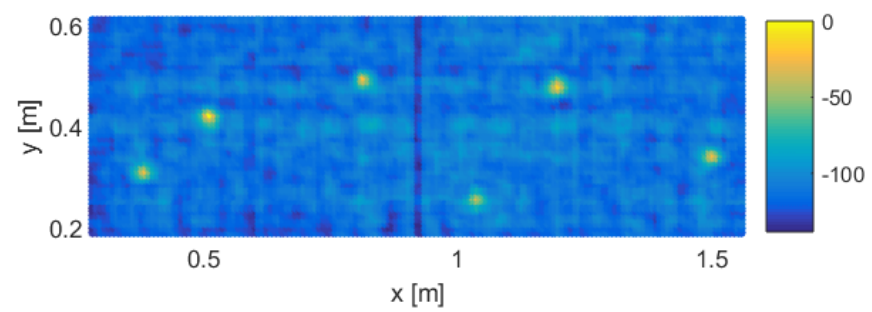

(d) Damage index based on $M=4=R_{0}^{\text {meas }}<R_{0}=6, N=11$ for 151 frequency lines between $500 \mathrm{~Hz}$ and $1000 \mathrm{~Hz}$. Logarithmic color scale.

Figure 2: Influence of undersapled data. 
At all frequencies, the measured signal space $S_{\Phi}^{\text {meas }}$ contributes information on the scatterers' locations, while the error is contributing arbitrarily at different frequencies. Therefore, in average over multiple frequencies, the product in equation (11) is no longer 0 , but still smaller than the one in equation (12). Applying equation (15) to this scenario allows to filter the coherent parts of the image between different frequencies and is thus a way to enable the detection of scatterers although the conditions required according to the theoretical derivation of the algorithm are not strictly fulfilled. The example for this case is presented in figure $2 \mathrm{~d}$, where all 6 scatterers are correctly indicated. The choice for $M=R_{0}^{\text {meas }}=4$ is somewhat arbitrary and should be understood as an illustration. The performance of the algorithm gradually increases the closer $S_{\Phi}^{\text {meas }}$ comes to $S_{\Phi}$, and hence depends on the choice of $R_{0}^{\text {meas }}$ compared to $R_{0}$.

A similar discussion treating the advantages of multi-frequency MUSIC (without an explicit link to TR) and in particular the interferometric MUSIC approach for the identification of scatterers in a high-frequency scenario, where there is no modal behaviour expected can be found in Solimene et al. [36]

In literature, multiple authors report on the trouble finding the correct separation between signal and noise space [20, 19]. Re-interpreting the findings presented in this section in the context of that problem, it follows for the same reasons that a good detection can still be achieved, although the separation between signal and noise space is slightly incorrect.

Furthermore, also the influence of random measurement noise on the detection quality decreases when using an interferometric TR-MUSIC algorithm as compared to a single frequency case. The reason for this is similar to what is explained so far in this section. The overlapping parts of the true and the measured signal space contribute coherent information of the defect location over all frequencies, while this is not the case for the erroneous contribution.

\subsection{Frequency selection for the TR-MUSIC algorithm}

As described in the previous section, adding multiple frequencies to the construction of the pseudospectrum has various advantages. However, if too many frequencies are included in the calculation of the denominator in equation (15) and the denominator for 
most of these frequencies is smaller than 1, numerical issues may arise, since equation (15) runs the risk of becoming infinite, even if $z$ is not a scatterer location. It is proposed to cope with this challenge by including only those frequency lines in the algorithm that add valuable information.

In order to evaluate whether the information carried at a specific frequency is valuable for a scatterer localisation based on equation [15], certain requirements must be fulfilled from experimental/measurement side $\left(\boldsymbol{\Phi}_{n}\right)$ as well as from the model's side $\left(\boldsymbol{g}_{f}(z)\right)$. All criteria used in this publication to distinguish between frequencies adding valuable information that should be kept and those frequencies that can be discarded are listed below and explained in more detail subsequently:

- Model: $\boldsymbol{g}_{f}(z)$ must be as different as possible for all $z$ investigated. This is because if $\boldsymbol{g}_{f}(z)$ was similar for all $z$, all $\boldsymbol{g}_{f}(z)$ would be close to orthogonal to $N_{\Phi}$. Therefore, even a small error would cause the product $\boldsymbol{g}_{f}(z)^{\mathrm{H}} \boldsymbol{\Phi}_{n}$ in the denominator of equation (15) to be comparable for all $z$, independent of the location of $z$. As such, no information would be added in this case.

- Measurement: The amplitude of the scattered wave field $\Psi_{s}\left(x_{n}, x_{m}\right)$ (equation (2)) should be sufficiently large to ensure that it can be measured under the given environmental conditions and with the measurement equipment in use.

315

In order to evaluate whether $\boldsymbol{g}_{f}(z)$ is sufficiently different for different $z$, first the MAC-value is calculated as [37]

$$
\operatorname{MAC}_{f}\left(z_{1}, z_{2}\right)=\frac{\left|\boldsymbol{g}_{f}\left(z_{1}\right)^{\mathrm{H}} \boldsymbol{g}_{f}\left(z_{2}\right)\right|^{2}}{\left|\boldsymbol{g}_{f}\left(z_{1}\right)^{\mathrm{H}} \boldsymbol{g}_{f}\left(z_{1}\right)\right|\left|\boldsymbol{g}_{f}\left(z_{2}\right)^{\mathrm{H}} \boldsymbol{g}_{f}\left(z_{2}\right)\right|} .
$$

$\operatorname{MAC}_{f}\left(z_{1}, z_{2}\right)$ is equal to 1 if the vectors $\boldsymbol{g}_{f}\left(z_{1}\right)$ and $\boldsymbol{g}_{f}\left(z_{2}\right)$ are equal and 0 if the two vectors are orthogonal. The values $\operatorname{MAC}_{f}\left(z_{1}, z_{2}\right)$ are collected in a lower triangular matrix $\boldsymbol{M} \boldsymbol{A} \boldsymbol{C}_{f}$ of dimension $Z \times Z$, where $Z$ is the number of potential scatterer locations 320 / pixels in the image $z_{n}$. Due to the symmetry of the MAC matrix, all the diagonal and upper triangular elements do not need to be calculated.

Desirable is a MAC matrix with as small as possible off-diagonal elements indicating 
that the vectors $\boldsymbol{g}_{f}\left(z_{n}\right)$ are in average as far as possible from being equal. This results in the first selection criterion, being: a frequency is included if

$$
\frac{\sum \boldsymbol{M A C _ { f }}}{\frac{(Z-1) Z}{2}}<M A C_{\max },
$$

325 threshold $M A C_{\max }$ must be set by the user.

Since $\boldsymbol{g}_{f}\left(z_{n}\right)$ is dependent on the sensor location and the location of potential scatterers $z$, also the question whether a frequency is included in the TR-MUSIC algorithm or not depends on these parameters. In NDT\&E applications, the potential object with scatterers and the vibration of the intact object, respectively, with dimension $1 \times N$. field, $\Psi_{s, f}$ is set in relation to the amplitude measured at the defected and the intact test 345
In order to be able to set a dimensionless threshold for the amplitude of the scattered

$$
\boldsymbol{\Psi}_{s, f}=\log \left(\left|\boldsymbol{\Psi}_{\text {meas }, f}-\boldsymbol{\Psi}_{\text {init }, f}\right|\right),
$$

where $\boldsymbol{\Psi}_{\text {meas }, f}$ and $\boldsymbol{\Psi}_{\text {init, } f}$ are vectors containing the vibration measured at the object:

$$
\Psi_{s, f}^{\text {norm }}=\frac{\boldsymbol{\Psi}_{s, f}}{\overline{\boldsymbol{R M} \boldsymbol{S}_{\Psi}}}
$$


with $\boldsymbol{\bullet}$ indicating the mean value and the matrix

$$
\boldsymbol{R M} \boldsymbol{S}_{\Psi}=\left[\begin{array}{ll}
R M S\left(\boldsymbol{\Psi}_{\text {meas }}\right) & R M S\left(\boldsymbol{\Psi}_{\text {init }}\right)
\end{array}\right]
$$

The abbreviation $R M S$ in equation (20) indicates the calculation of the root means square of a matrix, which is a positive, scalar value.

Based on the definitions of equation (19), a frequency is selected if the condition

$$
\frac{\sum_{N} \boldsymbol{\Psi}_{s, f}^{\text {norm }}}{N}>\Psi_{s, \min }
$$

350

with the user-set threshold $\Psi_{s, \min }$ is met.

Note that the selection of frequencies from sensor side is done separately for each excitation. Therefore, for small $M$, it is likely that $R_{0}^{\text {meas }}$ varies with frequency.

Frequency selection for the transmitter side usage of the TR-MUSIC algorithm can be derived similarly.

\subsection{TR-MUSIC with different sensor-types in one array}

As mentioned in the introduction, under certain conditions, it might be beneficial or simply necessary to combine data retrieved from sensors measuring different quantities. In principle, the derivation of the TR-MUSIC algorithm presented previously holds, independent of the quantities measured by the sensor array. However, if the values measured by different sensors are significantly different (orders of magnitude), the corresponding rows of $\boldsymbol{K}$ would be significantly larger than others. This can cause rank deficiency of $\boldsymbol{K}$ and as a consequence the dimension of $S_{\Phi}$ would decrease.

In order to avoid this happening, it is proposed to condition $\boldsymbol{K}$ according to the following routine. Again it is intended to facilitate reading by implicitly assuming that all variables and matrices in this subsection relate to one frequency.

First the sensors in the sensor array are grouped according to the physical quantity they measure. E.g. in the case of an array composed of accelerometers and microphones, these two types of sensors would form the two groups. Then the average Frobenius norm of each group is calculated: 


$$
\hat{q}_{k}=\frac{\left\|\left[\begin{array}{llll}
\boldsymbol{g}_{k}\left(z_{1}\right) & \boldsymbol{g}_{k}\left(z_{2}\right) & \ldots & \boldsymbol{g}_{k}\left(z_{Z}\right)
\end{array}\right]\right\|_{F r o}}{N_{k}},
$$
number of sensors in this group.

The matrix $\boldsymbol{K}$ is scaled by a left multiplication with the diagonal scaling matrix $\boldsymbol{B}$ containing the scaling factors $\hat{q}_{k}$ of equation (22) on the diagonal elements.

$$
\hat{\boldsymbol{K}}=\boldsymbol{B} \boldsymbol{K}=\left[\begin{array}{llll}
\operatorname{diag}\left(\hat{q}_{1}^{-1}\right) & & 0 & \\
& \operatorname{diag}\left(\hat{q}_{2}^{-1}\right) & & \\
& & \ddots & \\
0 & & \operatorname{diag}\left(\hat{q}_{k}^{-1}\right)
\end{array}\right] \boldsymbol{K} .
$$

As this change influences the singular vectors $\boldsymbol{\Phi}_{n}$, the vectors $\boldsymbol{g}(z)$ need to be adapted accordingly (proof in Appendix A):

$$
\hat{\boldsymbol{g}}(z)=\boldsymbol{B} \boldsymbol{g}(z)
$$

Although the physical meaning of $\boldsymbol{g}(z)$ gets partially lost due to this operation (e.g. it cannot be seen anymore that the acoustically radiated energy is a lot lower than the energy in the structural vibration due to the impedance mismatch), it allows to combine different sensor types, since the amplitude of each group of sensors in the array is now

${ }_{380}$ in a comparable range. Furthermore, additional freedom for the user is generated as no care needs to be taken to work in a consistent unit system between sensors of different groups.

The normalisation of $\boldsymbol{g}(z)$ as mentioned in Appendix B, should be done after this step.

385 There are two reasons to perform the conditioning of $\boldsymbol{K}$ based on $\boldsymbol{g}(z)$, rather than on the values in $\boldsymbol{K}$ itself. The first one is that $\boldsymbol{g}(z)$ needs to be measured only once for the intact structure. Therefore, without loosing time in the actual localisation process, this measurement can be repeated multiple times, ensuring a high reliability 
and at convenient moments, ensuring low-error measurements. Furthermore, since $\boldsymbol{g}(z)$ remains unchanged for all measurements at the defected structure, $\hat{q}_{k}$ can be computed a-priori, further minimising the computation time.

\section{Experimental validation}

In order to validate the developments of the previous section, a validation case with high complexity is considered. Furthermore, it is investigated whether a sensor array composed of sensors measuring different physical quantities can overcome limitations set by noisy measurements on one of the physical channels.

Therefore, first the measurement setup is explained, then the equations of the previous section are applied and artificial measurement errors are added on some sensors.

\subsection{Experimental setup}

The test object under investigation is an assembly of multiple components (figure 3). The base frame (figure $3 \mathrm{p}$ ) is composed of aluminium beams. Three vertically aligned beams with a C-cross-section and $4 \mathrm{~mm}$ wall thickness are joined with two horizontally aligned beams with I-cross-section and $3 \mathrm{~mm}$ wall thickness. The I-beams are constructed by merging two $\mathrm{C}$-sections with rivets. This construction results in six connection points $(Z=6)$ between the beams, where each connection is fixed with two bolts (figure 3 ). The frame is a replica of a section used in commercial air planes.

The I-section is covered by a honeycomb panel $(10 \mathrm{~mm}$ core thickness, skins on both sides with $0.3 \mathrm{~mm}$ thickness) of $1.4 \mathrm{~m} \times 0.5 \mathrm{~m}$. The connection between the honeycomb panel and the I-beam is realised via double-sided tape and bolts. Looking at the honeycomb panel, large parts of the frame, including all six connections are hidden. This view is referred to as 'front' in the following and all directions - top, bottom, left, right - are given with reference to this view.

The aim of this experiment is to find at which connection there is a defect, where a defect is simulated by loosening or removing bolts. In order to investigate if there is a dependency of the algorithm on the severity of a defect, different levels of severity are 


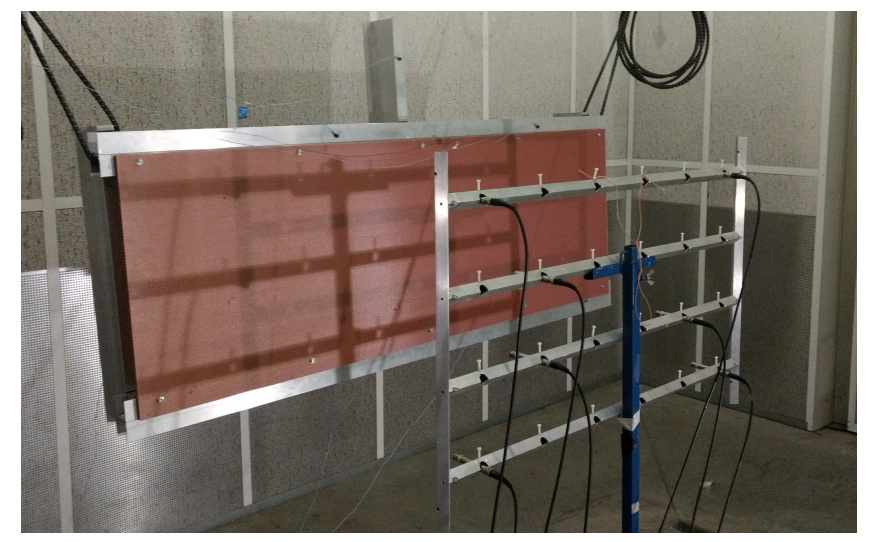

(a) Front view on the setup.

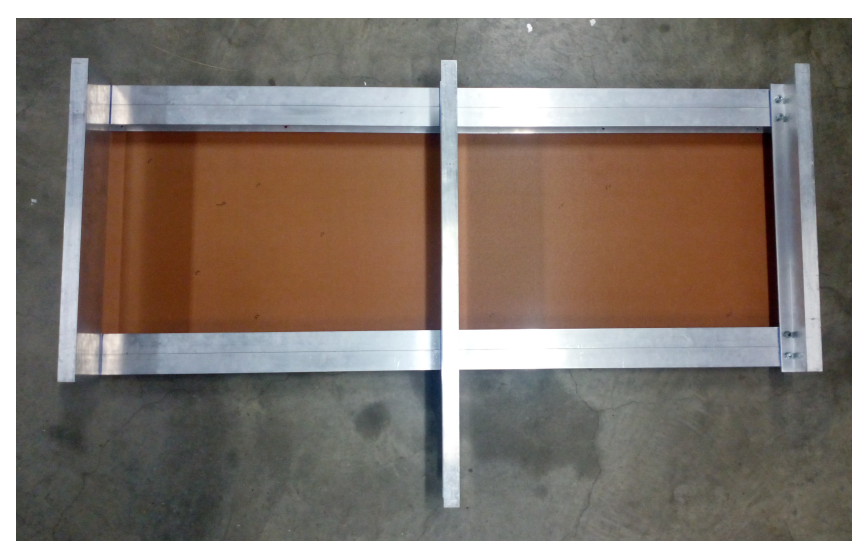

(b) Back view on test object.

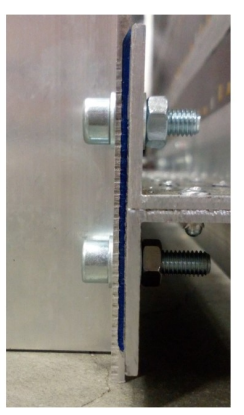

(c) Detail view on bottom right connection

Figure 3: Measurement setup, test object and detail view on one potential defect location. 

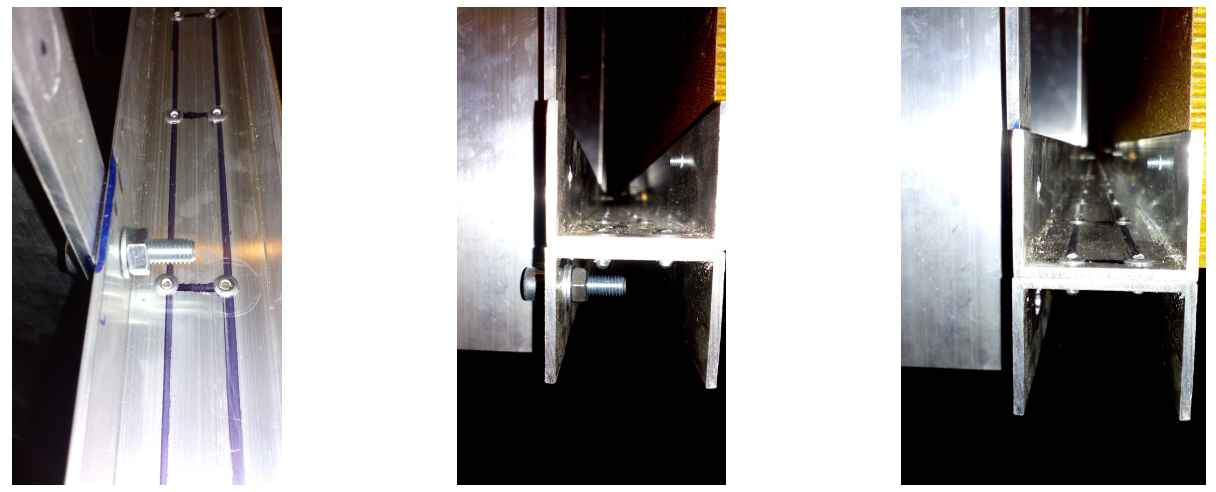

Figure 4: Example for one loose bolt (left), one removed bolt (centre) and two removed bolt (right).

introduced by either loosening one bolt at a connection or removing one or both bolts, respectively at the same connection. Whether the TR-MUSIC algorithm is able to point out defects at different locations is tested by loosening or removing bolts at different connections (multi-defect scenario). A complete list of all tested cases is given below:

1. One loose bolt at the top central connection (invisible with the bare eye, figure 4 left);

2. One removed bolt at the top central connection;

3. One loose bolt at the bottom left connection (invisible with the bare eye);

4. One removed bolt at the bottom left connection (figure 4 centre);

5. Two removed bolts at the bottom left connection (figure 4 right);

6. One loose bolt at the top right connection (invisible with the bare eye);

7. One removed bolt at the top right connection;

8. One removed bolt at the top right connection and one removed bolt at the top central connection.

For the localisation of these defects, in total $N=13$ sensors are installed, of which seven are microphones and six are 1-D accelerometers (PCB 352a24), measuring the out-of-plane displacement on the frame. All microphones (1/2", PCB 426E01 and Bruel \& Kjaer Type 2671) are installed in a plane at $0.5 \mathrm{~m}$ distance and parallel to the

${ }_{435}$ honeycomb panel. Three of the accelerometers are installed at the front side of the frame and three on the vertical C-sections. None of the defects is in the direct line of 
sight for any of the sensors. The test object is freely supported, so that the lower end is in a height of $0.9 \mathrm{~m}$ over the floor. The excitation is applied with an impact hammer (PCB 086C03) at $M=3$ locations on the front side of the frame. All experiments are 440 repeated 5 times and the results are averaged in order to suppress random measurement errors. The good repeatability of the experiment is verified by the comparison of two successive measurements in the beginning of the test campaign at the healthy structure and the measurement of the completely repaired structure at the end of the measurement campaign. The setup is installed in a semi-anechoic chamber (reflecting floor; absorbing walls and ceiling, designed for a cut-off frequency of $200 \mathrm{~Hz}$ ), due to its lower background noise level as compared to other areas in the lab. Other testsetups being installed simultaneously in the same semi-anechoic chamber cause that the assumption of a semi-free-field (full reflection at the floor; ideally radiation to infinity in all other directions) does not hold during the measurements, making the ${ }_{450}$ obtained results applicable to a more general environment. The measurement system is designed to capture a frequency range from $0 \mathrm{~Hz}$ to $900 \mathrm{~Hz}$ in steps of $0.5 \mathrm{~Hz}$. The actual measurement is acquired with a LMS SCADAS Mobile at a sampling frequency of $2048 \mathrm{~Hz}$ and triggered by the impact hammer.

It should be noted that due to the frequency selection procedure, at many frequency lines not all of the excitations are included in the algorithm, which is why $M=3$ is the maximum value and best possible case. At the same time, there are cases, where either defects are introduced at two different connections or both (all) bolts at one connection are removed. Certainly, both damage scenarios cannot be simplified by the assumption of a point scatterer. Therefore, in most of the cases studied, the data is undersampled ${ }_{460} \quad\left(R_{0}^{\text {meas }}=M<R_{0}\right)$.

The vectors $\boldsymbol{g}(z)$, needed to compute the pseudospectrum $I(z)$, are measured using the same equipment and settings as described in the paragraphs above. For the measurement of $\boldsymbol{g}(z)$ for one potential defect location $z$, the respective bolt head is excited with an impact hammer-hit in direction of the bolt's symmetry axis and the response is 465 measured by all sensors. The resulting frequency response function is used to define $g(z)$. 


\subsection{Localisation under ideal conditions}

In this section only the results for the localisation of defect cases 1, 5, 7 and 8 based on the actually measured data are presented in detail. Since the outcome of the localisation of all other defects is in accordance with what is presented and all conclusions apply, the other 4 cases are only presented in an overview to avoid redundancy. The localisation results for these four cases are shown in figure 5 On the $\mathrm{x}$-axis, the potentially defected connections are listed, where TL refers to the top left connection, BL to the bottom left connection and so on. In the figures at the left, for each connection, the calculated pseudospectrum $I(z)$ normalised by the largest $I(z)$ is presented, so that the y-axis ranges from 0 to 1 . The figures at the right illustrate the logarithm of the left figures.

Especially when analysing the figures at the left, it is apparent that in all cases, one location is uniquely highlighted as defected. This includes all different types of defects introduced at only one connection (figure $5 \mathrm{a}-\mathrm{c}$ ), which are uniquely highlighted by the TR-MUSIC pseudospectrum at the correct location. This observation demonstrates that the developed algorithm is a strong tool for the position determination of a variety of defects, even in complex assemblies.

Figure $5 \mathrm{~d}$ shows the result for defects at two different connections. Clearly only one of these connections is correctly highlighted, but uniquely, which means that also in this case no false positive detection is given. Furthermore, after fixing the highlighted connection and repeating the test in this condition, only the second defected connection is indicated by the pseudospectrum. Therefore, it can be concluded that the TR-MUSIC algorithm as defined in this publication is not able to locate multiple defects at once. Nevertheless this example indicates the potential of the algorithm for the sequential determination of multiple defects.

The large difference of multiple orders of magnitude between the pseudospectrum at the defected location and at other locations comes to the fore at the graphs at the right in figure 5

In figure 6, the detection results for all 8 defect locations using different settings and sensor arrays are presented. On the x-axis, the different defect cases $1-8$ are listed in accordance with the list given in the section 'Experimental setup]. Each number on 

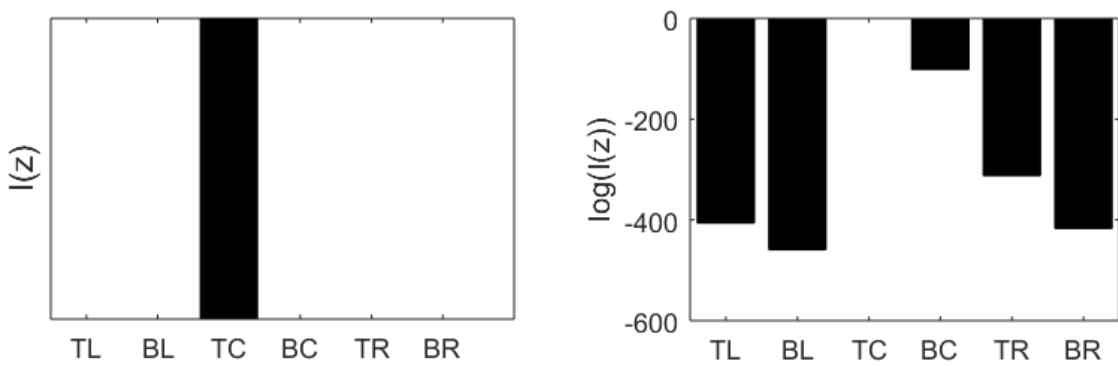

(a) One loose bolt at the top central connection (case 1).
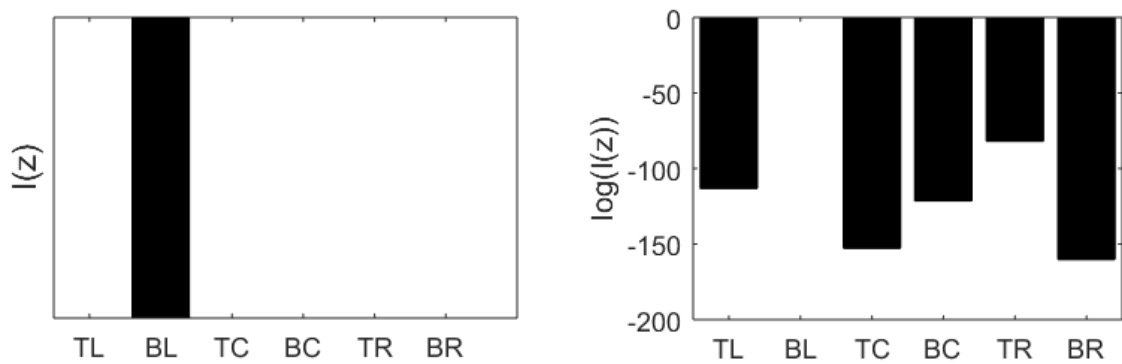

(b) Two removed bolts at the bottom left connection (case 5).
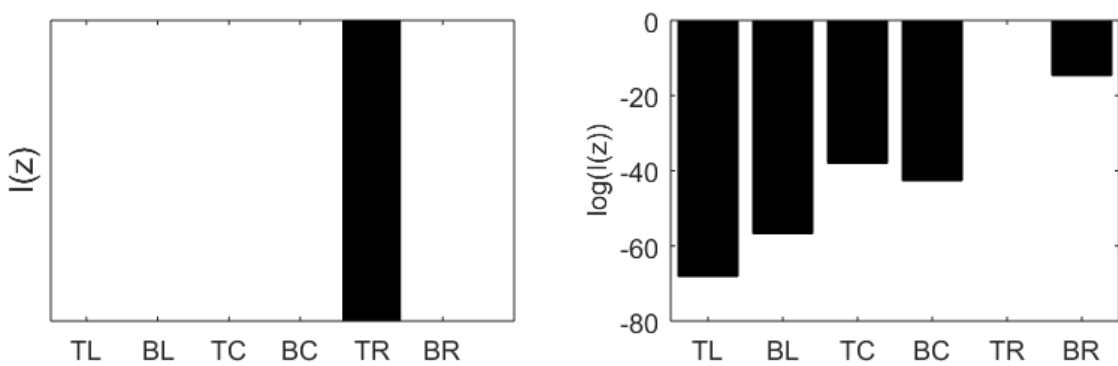

(c) One removed bolt at the top right connection (case7).
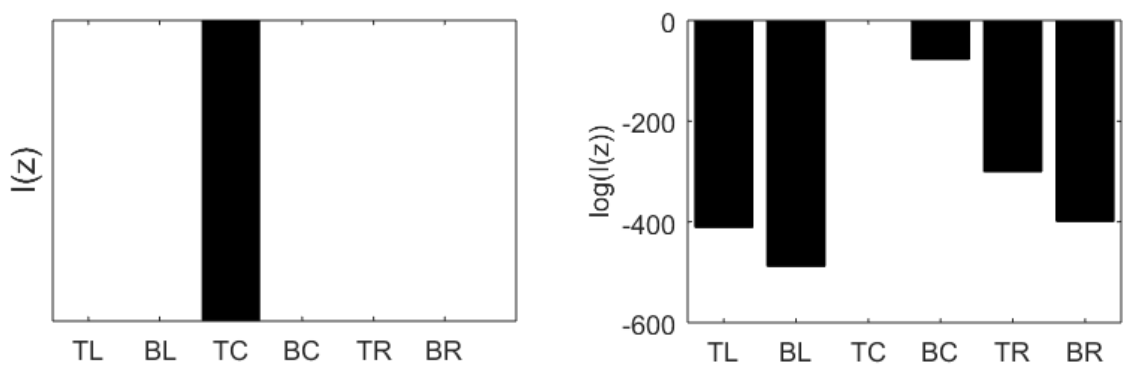

(d) One removed bolt at the top right connection and one removed bolt at the top central connection (case 8).

Figure 5: Normalised pseudospectrum $I(z)$ for all six connections (left) and the logarithmic illustration $\log (I(z))$ (right). 


\begin{tabular}{c|c|c|c} 
Setup number & Selection criterion & Sensors & Scaling \\
\hline \hline 1 & $M A C_{\text {max }}, \Psi_{s, \text { min }}$ & all & yes \\
\hline 2 & $M A C_{\max }, \Psi_{s, \text { min }}$ & Mic. & not applicable \\
\hline 3 & $M A C_{\max }, \Psi_{s, \text { min }}$ & Accel. & not applicable \\
\hline 4 & $M A C_{\max }, \Psi_{s, \text { min }}$ & all & no \\
\hline 5 & $\Psi_{s, \min }$ & all & yes \\
\hline 6 & $M A C_{\max }$ & all & yes
\end{tabular}

Table 1: Overview of setup configurations for the results presented in figure 6

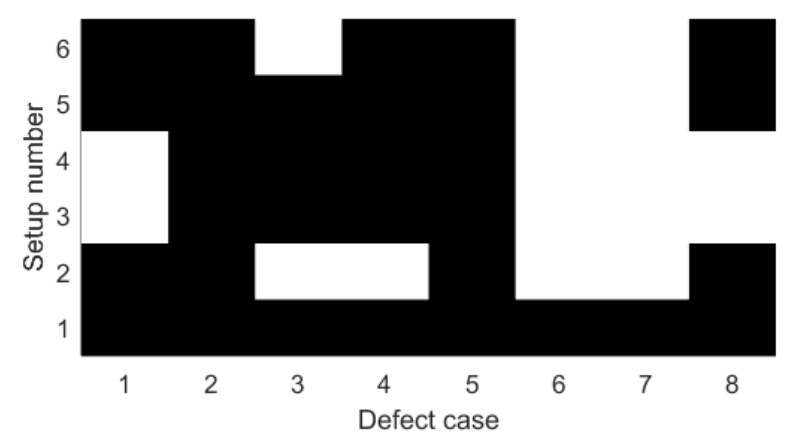

Figure 6: Localisation results for 8 different defect cases, 6 different configurations (see table 1 ) and $\vartheta=100$. Black indicates successful localisation, white indicates a failed localisation.

the $y$-axis represents one of the setup configurations listed in table 1 . A localisation is assessed as 'successful', if the pseudospectrum at the correct location is at least a factor $\vartheta$ higher than the pseudospectrum at any other location (due to the previous discussion, case 8 is also regarded as 'located successfully' if only one of the two defects is highlighted). Successful localisations are indicated with a black field, failed ones with a white field.

As already indicated at the beginning of this section, if all described selection criteria and the scaling for different sensor types is applied to an array composed of microphones and accelerometers, the localisation of all defects under investigation is possible (figure 3 , setup 1). If the array is built by only microphones (setup 2) or only accelerometers (setup 3), respectively, then not all defects can be located correctly. 
Here, it should be noted that the size of a purely structural or acoustic array is smaller than that of a combined array. Also if the scaling for different sensor types (setup 4) or one of the selection criteria (setup 5 and 6) are not applied, the localisation fails underlining the necessity of all previously described steps.

\subsection{Localisation in noisy environment}

As demonstrated in the previous section, the TR-MUSIC algorithm reliably locates defects based on data measured in an environment with low background noise. However, such close to ideal measurement conditions rarely exist in real-life measurements. In many test environments, e.g. outdoor or in a production plant, especially the pollution of the data measured by acoustic sensors with ambient noise is not uncommon. Therefore, in this section the robustness of the algorithm in face of measurement errors acting mainly on one group of sensors is investigated.

In order to perform this investigation, artificial, random measurement noise with zero mean is added on the measured data. The singular value decomposition of $\boldsymbol{K}$ in the TR-MUSIC algorithm is then replaced by the decomposition of $\boldsymbol{K}^{\epsilon}$ containing the erroneous measurement of the scattered field. It is calculated as:

$$
\boldsymbol{K}^{\boldsymbol{\epsilon}}=\boldsymbol{K}+\left[\begin{array}{llll}
\boldsymbol{\epsilon}_{1}^{\mathrm{T}} \sqrt{\beta_{1}} & \boldsymbol{\epsilon}_{2}^{\mathrm{T}} \sqrt{\beta_{2}} & \cdots & \boldsymbol{\epsilon}_{K}^{\mathrm{T}} \sqrt{\beta_{K}}
\end{array}\right]^{\mathrm{T}},
$$

where $\beta_{k}$ is a scalar factor defining the amplitude of the artificially applied error on the group of sensors $k$ and $\boldsymbol{\epsilon}$ is a matrix of the same dimension as $\boldsymbol{K}$ but filled with random complex numbers following a standard normal distribution. Correspondingly, $\boldsymbol{\epsilon}_{k}$ refers to the error added to the k-th group of sensors. $\beta_{k}$ depends on the requested error level per group of sensors $S N R_{k}$ in the following way:

$$
\beta_{k}=\frac{\sum_{F}\left|\boldsymbol{K}_{f, k}\right|^{2}}{10^{\frac{S N R_{k}}{10}} \sum_{F}\left|\boldsymbol{\epsilon}_{\boldsymbol{f}, \boldsymbol{k}}\right|^{2}} .
$$

As can be seen from equation (26), when $S N R_{k}$ tends towards infinity, $\beta_{k}$ tends to 0 , which means that there is no error added to the respective group of sensors. 
In order to give a tangible impression on the meaning of the $S N R$ values, the frequency response function measured by one representative microphone at defect case 5 is shown in figure 7. The plot at the bottom is a representation of the scattered wave field, computed according to equation (2), with noise added according to different $S N R$ values. This is the quantity, which is directly used by the algorithm. The top of figure 7 shows the same absolute errors, now added to the pressure directly measured by the microphone. This comparison is of interest, since here the influence of measurement noise in relation to the actually measured quantity is visualised. In both cases it can be seen that $S N R=20$ and $S N R=10$ represent significant measurement errors. In the case of $S N R=10$, even in the frequency response function of the measurement at the defected test object, apart from the resonances, only noise, no signal pattern can be seen.

In tables 2 to 5 , the detection results for all 8 defect locations under different error conditions are presented. For each case, the simulation is repeated 100 times with the same $S N R$ value applied to the same group of sensors. However, due to the random nature of the error, results are not necessarily equal. In each table, the number of correct localisations is presented in the first content row. It should be emphasised that the term 'correct' depends on the choice of $\vartheta$, which is given in the table caption. Furthermore, in each table, the number of simulations, where none of the connections is highlighted as defected and if applicable the number of false positive localisations is shown. The sum of each column individually equals 100 , the total number of simulations.

Tables 2 and 3 show results, where the artificial error is only applied to the microphone signals, while the measurement values provided by the accelerometers remain untouched. In table $2 \mathrm{a}$, it can be seen that even with $S N R_{\text {mic }}=20$ in most simulations, the defect is correctly localised applying a factor of $\vartheta=100$. In this scenario, the algorithm only struggled in 65 simulations with the localisation of a defect at the top right position (cases 6 and 7), while in 735 simulations the localisation worked successfully. Increasing the error level to $S N R_{m i c}=10$ (table 2 $\mathrm{p}$ ), still the defects at the majority of the locations are located correctly. With $S N R_{m i c}=10$, still all defects at the top centre (case 1 and 2) and at the bottom left (case 3,4 and 5) are always located correctly, but the number of false localisations for defects at the top right position increased to 

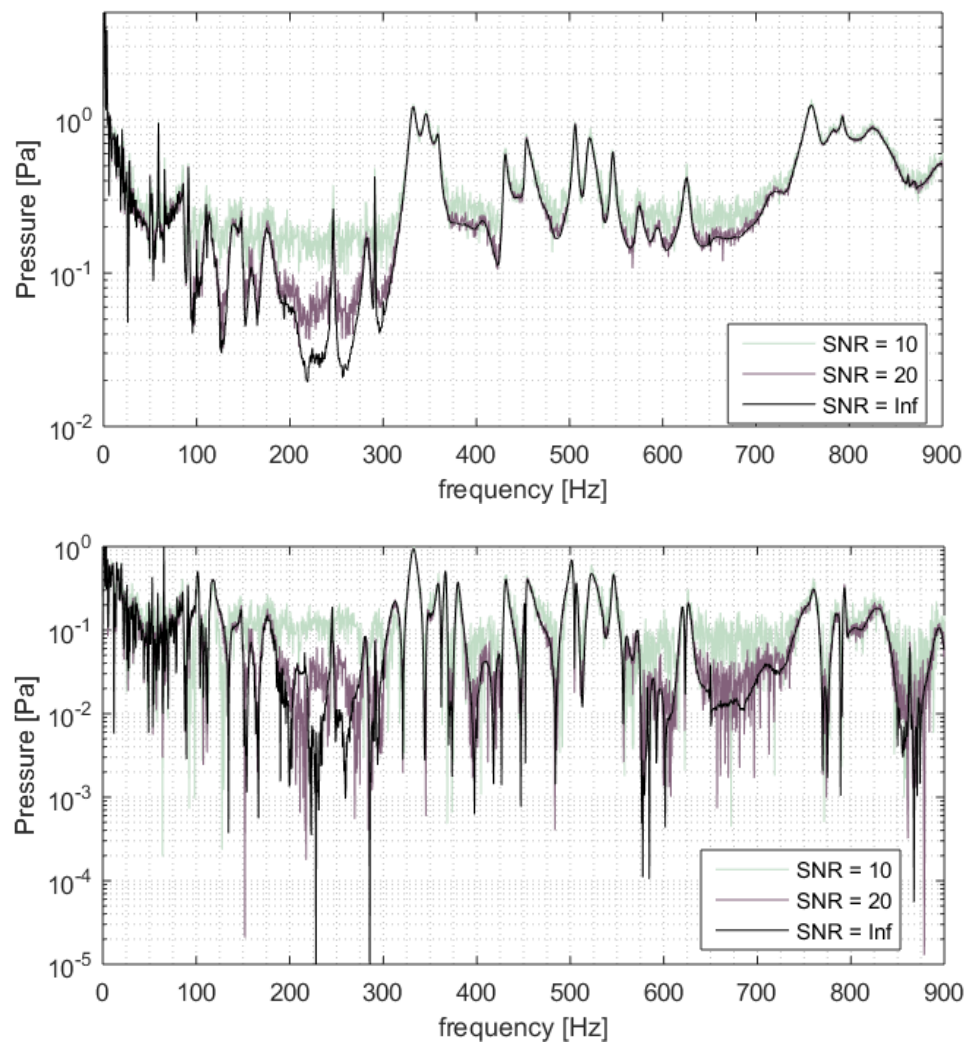

Figure 7: Frequency response function at one microphone with different artificial error levels. Top: influence of the error on the pressure measured directly at the defect case 5. Bottom: The influence of the error on the corresponding scattered field. 


\begin{tabular}{c|c|c|c|c|c|c|c|c} 
Defect case & 1 & 2 & 3 & 4 & 5 & 6 & 7 & 8 \\
\hline \hline Correct localisation & 100 & 100 & 100 & 100 & 100 & 36 & 99 & 100 \\
\hline No unique localisation & & & & & & 64 & 1 &
\end{tabular}

(a) $S N R_{\text {mic }}=20, S N R_{a c c}=\operatorname{Inf}$

\begin{tabular}{c|c|c|c|c|c|c|c|c} 
Defect case & 1 & 2 & 3 & 4 & 5 & 6 & 7 & 8 \\
\hline \hline Correct localisation & 100 & 100 & 100 & 100 & 100 & 27 & 77 & 100 \\
\hline No unique localisation & & & & & & 73 & 23 &
\end{tabular}

(b) $S N R_{\text {mic }}=10, S N R_{a c c}=\operatorname{Inf}$

Table 2: Localisation results for 8 different defect cases and 100 repetitions with different noise levels added to the microphones. The threshold for a successful localisation is set at $\vartheta=100$.

\begin{tabular}{c|c|c|c|c|c|c|c|c} 
Defect case & 1 & 2 & 3 & 4 & 5 & 6 & 7 & 8 \\
\hline \hline Correct localisation & 100 & 100 & 100 & 100 & 100 & 99 & 100 & 100 \\
\hline No unique localisation & & & & & & 1 & &
\end{tabular}

(a) $S N R_{\text {mic }}=20, S N R_{a c c}=\operatorname{Inf}$

\begin{tabular}{c|c|c|c|c|c|c|c|c} 
Defect case & 1 & 2 & 3 & 4 & 5 & 6 & 7 & 8 \\
\hline \hline Correct localisation & 100 & 100 & 100 & 100 & 100 & 74 & 99 & 100 \\
\hline False positive at BR & & & & & & 5 & & \\
\hline No unique localisation & & & & & & 21 & 1 &
\end{tabular}

(b) $S N R_{m i c}=10, S N R_{a c c}=\operatorname{Inf}$

Table 3: Localisation results for 8 different defect cases and 100 repetitions with different noise levels added to the microphones. The threshold for a successful localisation is set at $\vartheta=2$.

96. Since the amplitude of the scattered wave field for defects at this position is lower than for defects at any other connection, it is assumed that due to the placement of excitations and sensors defects at the top right are more difficult to detect than defects at other places and thus the TR-MUSIC algorithm fails first for the localisation of defects at this location. As a consequence, it is expected that the localisation of defects at the top right could be more accurate by adding sensors and excitations or by changing their 
location. As mentioned previously, the optimal placement of sensors and excitations is

for $S N R_{\text {mic }}=10$ (table 3p), it can be seen that the number of correct detection improves as compared to table $2 \mathrm{p}$, but still in 27 cases a defect at the top right location is not found. In all these cases, the reason is a too large pseudospectrum at the bottom right location, resulting in either a false positive localisation of the bottom right or in 580 a scenario, where no unique localisation is possible because the pseudospectrum at the bottom right and at the top right are approximately equal. This observation can be explained, as the factor between the pseudospectrum at the correctly pointed out top right and that at the bottom right under perfect measurement conditions is 4421 and as such many orders of magnitude smaller than the factor to other connections $\left(10^{17}\right.$ and higher). Perturbing parts of the measurement further decreases that factor and leads to false detections first, where the difference is smallest.

While in tables 2 and 3 the artificial measurement error was only added to the microphones, in tables 4 and 5 , the same levels of measurement error are added to all sensors. The conclusions that can be drawn for an error level of $S N R=20$ (tables 4 p and 3 for both error levels. If the highest error level under investigation of $S N R=10$ is applied to all sensors (tables $4 \mathrm{~b}$ and $5 \mathrm{~b}$ ), the localisation of a loose bolt at the bottom left connection (case 4) becomes the most challenging case. This statement is only weakly dependent on the chosen value for $\vartheta$, as can be seen in the comparison of table 595 4b with $\vartheta=100$ and table $5 \mathrm{~b}$ with $\vartheta=2$. It follows that most localisations did not fail due to an insufficiently high difference between the pseudospectrum at the bottom left and another connection, but rather due to a false positive localisation of an non-defected connection. The reason for this and the seeming discrepancy to the results shown in table 4 are explained in the following paragraphs. 


\begin{tabular}{c|c|c|c|c|c|c|c|c} 
Defect case & 1 & 2 & 3 & 4 & 5 & 6 & 7 & 8 \\
\hline \hline Correct localisation & 100 & 100 & 100 & 100 & 100 & 17 & 100 & 100 \\
\hline No unique localisation & & & & & & 83 & &
\end{tabular}

(a) $S N R_{\text {mic }}=20, S N R_{a c c}=20$

\begin{tabular}{c|c|c|c|c|c|c|c|c} 
Defect case & 1 & 2 & 3 & 4 & 5 & 6 & 7 & 8 \\
\hline \hline Correct localisation & 100 & 100 & 100 & 33 & 100 & 100 & 100 & 100 \\
\hline False positive at TR & & & & 12 & & & & \\
\hline No unique localisation & & & & 55 & & & &
\end{tabular}

(b) $S N R_{\text {mic }}=10, S N R_{a c c}=10$

Table 4: Localisation results for 8 different defect cases and 100 repetitions with different noise levels added to all sensors. The threshold for a successful localisation is set at $\vartheta=100$.

\begin{tabular}{c|c|c|c|c|c|c|c|c} 
Defect case & 1 & 2 & 3 & 4 & 5 & 6 & 7 & 8 \\
\hline \hline Correct localisation & 100 & 100 & 100 & 100 & 100 & 87 & 100 & 100 \\
\hline False positive at BR & & & & & & 2 & & \\
\hline No unique localisation & & & & & & 11 & &
\end{tabular}

(a) $S N R_{\text {mic }}=20, S N R_{\text {acc }}=20$

\begin{tabular}{c|c|c|c|c|c|c|c|c} 
Defect case & 1 & 2 & 3 & 4 & 5 & 6 & 7 & 8 \\
\hline \hline Correct localisation & 100 & 100 & 100 & 63 & 100 & 100 & 100 & 100 \\
\hline False positive at TR & & & & 28 & & & & \\
\hline No unique localisation & & & & 9 & & & &
\end{tabular}

(b) $S N R_{\text {mic }}=10, S N R_{\text {acc }}=10$

Table 5: Localisation results for 8 different defect cases and 100 repetitions with different noise levels added to all sensors. The threshold for a successful localisation is set at $\vartheta=2$.

Looking closer at these results, it is observed that every false detection in tables $4 \mathrm{p}$ and $5 \mathrm{p}$ highlights the top right connection instead of the bottom left. This can be explained by analysing the vectors $\boldsymbol{g}(z)$. Summing the absolute values of all entries of $\boldsymbol{g}(z)$ over all frequencies, the value obtained at $z=$ 'bottom left' is slightly smaller than 
the values for the other connections. Since at this high noise level the separation between

of the scattered field for defects at this connection are actually lowest.

Depending on the choice of $\vartheta$, the number of unique localisations changes. At small values of $\vartheta$, only a small difference between the highest pseudospectrum and the pseudospectrum computed for other potential defect locations is required to classify a 630 as well the number of false positive localisations increases with decreasing $\vartheta$. On the other hand, the higher $\vartheta$, the more reliable is a localisation result. The comparison of table $2 \mathrm{p}$ with $3 \mathrm{~b}$, table $4 \mathrm{a}$ with $5 \mathrm{a}$ and table $4 \mathrm{~b}$ with $5 \mathrm{p}$ support this expectation.

The results presented in this section show that the localisation of defects provided by 
to heavy noise. Furthermore, it can be stated that the localisation with measurement errors on only one group of sensors in the array is superior to a scenario, where all sensors are exposed to noise. For the vibro-acoustic array with erroneous microphone measurements, at the error level of $S N R_{\text {mic }}=20$ still a reliable localisation is obtained

${ }_{640}$ (with difficulties to localise one loose bolt at the top right location), while for $S N R_{m i c}=$ 10 the procedure for more cases in this example starts to fail. However, with reference to table 7, even the noise level $S N R_{\text {mic }}=20$ is a severe perturbation of the correct measured signal. In conclusion, the initial assumption 'a vibro-acoustic array can be used in environments with high background noise' can be confirmed.

Also the claim of a speed-up of the overall test time seems plausible since the test was performed using only an impact hammer, seven microphones and six accelerometers. An additional advantage could be that this equipment is low priced as compared to highly specialised excitation systems in combination with a huge number of sensors, Laser-vibrometers or IR-cameras as they are in use for other inspection techniques. at only three excitation locations and the fact that the microphones do not need to be physically attached to the test object leads to further benefits in time.

\section{Conclusion}

In order to contribute to the development of a NDT\&E system for the automated inspection of complex mass produced products, it is proposed to use a TR-MUSIC algorithm in combination with a vibro-acoustic sensor-array composed of microphones and enhanced by structural sensors. A proposal on how to handle different sensor types in one array is described in this publication. Using such a setup in combination with impact-hammer excitation, the shortcomings of traditional technologies, such as object or the sensitivity of purely acoustic arrays to ambient noise can be overcome.

It is, furthermore, shown how to further improve the inspection time by reducing the number of excitations. It is demonstrated that the number of excitations can be 
pushed even below the theoretically necessary limit. In addition, a strategy to select the robustness and quality of the localisation in simple application cases and to enable defect localisation in complex structures.

The proposed method is experimentally applied to the detection of defects in a structure of industrial complexity, composed of aluminium bars, which are connected by bolts and rivets. One side of the test object is covered with a honeycomb panel, so that the bolts are not visible for the sensor array. The structure is excited one after another at three locations with an impact hammer and the vibrations are measured by an array of six 1-D accelerometers and seven microphones. The experiment showed that the TR-MUSIC algorithm is able to locate defects of different severity at different

\section{Acknowledgements}

The European Commission is gratefully acknowledged for their support of the ANTARES project (GA606817). Elke Deckers is a postdoctoral fellow of the Research Foundation - Flanders (FWO). Also the Research Fund KU Leuven is gratefully acknowledged for its support. Furthermore, the authors gratefully acknowledge SIM Flanders Innovation \& Entrepreneurship) for their support of the SBO project DETECTIV, which is part of the research program MacroModelMat (M3). 


\section{Appendix A: Proof for retained orthogonality of singular vectors after scaling}

For the TR-MUSIC algorithm, the orthogonality between signal and noise space is used. But since the signal and noise space spanned by the singular vectors of $\boldsymbol{K}$ is not necessarily equal to that spanned by the singular vectors of $\hat{\boldsymbol{K}}$, proof needs to be given that the scaling done in equations (23) and 24) preserves the required orthogonality between the the noise space resulting from the singular value decomposition of $\hat{\boldsymbol{K}}$ and the test vector $\boldsymbol{g}(z)$ for a point $z$ on a scatterer's surface.

Assumed is a matrix $\boldsymbol{A}$, which can be decomposed into its singular vectors and values

$$
\boldsymbol{A}=\boldsymbol{U} \boldsymbol{S} \boldsymbol{V}^{\mathrm{H}},
$$

where $\boldsymbol{U}$ and $\boldsymbol{V}$ are square matrices, whose columns are orthonormal vectors and $\boldsymbol{S}$ is a matrix containing the singular values on its diagonal elements.

Logically, the matrix $\boldsymbol{A}$ scaled with the diagonal scaling matrix $\boldsymbol{B}$ writes as

$$
\boldsymbol{B} \boldsymbol{A}=\boldsymbol{B} \boldsymbol{U} \boldsymbol{S} \boldsymbol{V}^{\mathrm{H}}=\boldsymbol{U}_{\text {scaled }} \boldsymbol{S}_{\text {scaled }} \boldsymbol{V}_{\text {scaled }}^{\mathrm{H}}
$$

Decomposing the product $\boldsymbol{B} \boldsymbol{U}$ into the product of an orthogonal matrix $\boldsymbol{Q}$ and an upper triangular matrix $\boldsymbol{R}$ applying a $\mathrm{QR}$ factorisation and introducing this into equation A.2) results in

$$
\boldsymbol{B} \boldsymbol{A}=\boldsymbol{Q R S} \boldsymbol{V}^{\mathrm{H}}
$$

Writing $R S$ as the product of its singular values and vectors $U_{1} S_{1} V_{1}^{\mathrm{H}}$ gives

$$
\boldsymbol{B} A=\boldsymbol{Q} \boldsymbol{U}_{1} \boldsymbol{S}_{1} \boldsymbol{V}_{1}^{\mathrm{H}} \boldsymbol{V}^{\mathrm{H}}
$$

Since the product of two unitary matrices gives another unitary matrix (if $\boldsymbol{Q}^{\mathrm{H}} \boldsymbol{Q}=\boldsymbol{I}$ and $\boldsymbol{U}_{1}^{\mathrm{H}} \boldsymbol{U}_{1}=\boldsymbol{I}$, then $\left.\left(\boldsymbol{Q} \boldsymbol{U}_{1}\right)^{\mathrm{H}}\left(\boldsymbol{Q} \boldsymbol{U}_{1}\right)=\boldsymbol{U}_{1}^{\mathrm{H}} \boldsymbol{Q}^{\mathrm{H}} \boldsymbol{Q} \boldsymbol{U}_{1}=\boldsymbol{U}_{1}^{\mathrm{H}} \boldsymbol{I} \boldsymbol{U}_{1}=\boldsymbol{I}\right)$, it follows that $\boldsymbol{Q} \boldsymbol{U}_{1}$ 
and $\boldsymbol{V}_{1}^{\mathrm{H}} \boldsymbol{V}^{\mathrm{H}}$ are the unitary matrices. Comparing equations $\mathrm{A} .2$ and $\mathrm{A} .4$, , it can be concluded that the columns of $\boldsymbol{Q} \boldsymbol{U}_{1}$ are orthogonal to the columns of $\boldsymbol{U}_{\text {scaled }}$ and the rows of $\boldsymbol{V}_{1}^{\mathrm{H}} \boldsymbol{V}^{\mathrm{H}}$ are orthogonal to the rows of $\boldsymbol{V}_{\text {scaled }}^{\mathrm{H}}$. However, since it cannot be guaranteed that $S_{1}=S_{\text {scaled }}$, no statement can be made regarding the orthogonality between the rows of $\boldsymbol{Q} \boldsymbol{U}_{1}$ and $\boldsymbol{U}_{\text {scaled }}$ or the orthogonality between the columns of $\boldsymbol{V}_{1}^{\mathrm{H}} \boldsymbol{V}^{\mathrm{H}}$ and $\boldsymbol{V}_{\text {scaled }}^{\mathrm{H}}$. This relation holds for all $\boldsymbol{K} \in \mathbb{C}$.

Since a basic assumption of the TR-MUSIC algorithm is the rank deficiency of the data matrix, in the following, rank deficiency of $\boldsymbol{A}$ is assumed, so that the singular values can be split into signal and noise space. In order to prove that the necessary orthogonality is preserved after the scaling, it needs to be shown that the noise space singular vectors of $\boldsymbol{U}_{\text {scaled }}$ are orthogonal to the signal space singular vectors of $\boldsymbol{B} \boldsymbol{U}$.

This orthogonality relation can be written as

$$
\boldsymbol{U}_{\text {scaled }}^{\mathrm{H}} \boldsymbol{Q} \boldsymbol{U}_{1}=\boldsymbol{D}
$$

where $\boldsymbol{D}$ is a diagonal matrix.

Introducing the relation previously given by the QR-factorisation of $\boldsymbol{B} \boldsymbol{U}$ and the singular value decomposition of $\boldsymbol{R S}$ into equation A.6 results in:

$$
\boldsymbol{U}_{\text {scaled }}^{\mathrm{H}} \boldsymbol{B} \boldsymbol{U}=\boldsymbol{D} \boldsymbol{S}_{1} \boldsymbol{V}_{1}^{\mathrm{H}} \boldsymbol{S}^{-1}
$$

In the following, it is assumed, that the singular vectors are ordered such that the signals space singular vectors are first, followed by the noise space singular vectors $\left(\boldsymbol{U}_{\bullet}=\left[\boldsymbol{U}_{\bullet}^{\text {signal }} \boldsymbol{U}_{\bullet}^{\text {noise }}\right]\right.$ and correspondingly for all other vectors $)$. Due to the rank deficiency, the product $S_{1} V_{1}^{\mathrm{H}}$ has only non-zero entries in the elements corresponding 730 to the signal space

$$
\boldsymbol{S}_{1} \boldsymbol{V}_{1}^{\mathrm{H}}=\left[\begin{array}{cc}
\operatorname{diag}\left(\boldsymbol{P}_{1}\right) & \mathbf{0} \\
\mathbf{0} & \mathbf{0}
\end{array}\right]\left[\begin{array}{cc}
\boldsymbol{P}_{2} & \mathbf{0} \\
\mathbf{0} & \boldsymbol{N}_{1}
\end{array}\right]=\left[\begin{array}{cc}
\boldsymbol{P}_{3} & \mathbf{0} \\
\mathbf{0} & \mathbf{0}
\end{array}\right],
$$

where $\boldsymbol{P}_{n}$ and $\boldsymbol{N}_{n}$ indicate matrices relating to the signal or noise space, respectively. 
Equation A.7 shows that the desired orthogonality between signal and noise space is not violated due to the product $S_{1} V_{1}^{\mathrm{H}}$.

The inverse of the last element of the right hand side of equation A.6 $S^{-1}$ is not defined due to the rank deficiency of $\boldsymbol{A}$ and the consequential full-zero rows and columns in $\boldsymbol{S}$. However, since the diagonal of the signal space of matrix $S$ is fully populated, also its inverse is uniquely defined, linking the signal space singular vectors of $\boldsymbol{U}$ and $\boldsymbol{V}$ and being independent of the noise space components. Therefore, some elements of $S^{-1}$ are known and the product

$$
\boldsymbol{D} \boldsymbol{S}_{1} \boldsymbol{V}_{1}^{\mathrm{H}} \boldsymbol{S}^{-1}=\boldsymbol{D}\left[\begin{array}{cc}
\boldsymbol{P}_{3} & \mathbf{0} \\
\mathbf{0} & \mathbf{0}
\end{array}\right]\left[\begin{array}{cc}
\operatorname{diag}\left(\boldsymbol{P}_{4}\right) & ? \\
\mathbf{0} & ?
\end{array}\right]=\boldsymbol{D}\left[\begin{array}{cc}
\boldsymbol{P}_{5} & ? \\
\mathbf{0} & ?
\end{array}\right]=\left[\begin{array}{cc}
\boldsymbol{P}_{6} & ? \\
\mathbf{0} & ?
\end{array}\right]
$$

can be partially defined. Although this operation is not fully defined, the $\mathbf{0}$ in the lower left of equation A.8 is sufficient to prove that the noise space singular vectors of $\boldsymbol{U}_{\text {scaled }}$ are orthogonal to the signal space singular vectors of $\boldsymbol{B} \boldsymbol{U}$.

\section{Appendix B: Normalisation of test vectors}

The vectors $\boldsymbol{g}_{f}(z)$, contain the transfer functions connecting the scatterer(s) to the sensors. In the low and mid frequency region, these test vectors can have very different amplitudes for different points $z \in D$ due to nodal points, lines and maxima of the dominating modes.

Errors in the measured matrix $\boldsymbol{K}$ directly lead to an error in the singular vectors $\boldsymbol{\Phi}_{n}$. In the following, the influence of such measurement errors is explained at the example of a sensor side defect localisation. However, what is described holds directly also for the computation of the pseudospectrum from excitation side by replacing all parameters belonging to the sensor side with their counter parts from the excitation side (e.g. replace $\boldsymbol{\Phi}_{n}$ with $\boldsymbol{\Psi}_{n}$ ).

Most obvious is the influence of errors, when only a single frequency is considered. 
Then equation 13 changes to

$$
\begin{aligned}
I(z) & =\left(\left|\boldsymbol{g}(z)^{H}\left(\boldsymbol{\Phi}_{n}+\boldsymbol{\epsilon}\right)\right|\right)^{-1} \\
& =\left(\left|\boldsymbol{g}(z)^{H} \boldsymbol{\Phi}_{n}+\boldsymbol{g}(z)^{H} \boldsymbol{\epsilon}\right|\right)^{-1},
\end{aligned}
$$

where the erroneous change of $\boldsymbol{\Phi}_{n}$ is expressed by the addition of the random error vector $\epsilon$. For equation B.1 and all following expressions relating to the pseudospectra, it is implicitly assumed that $n$ is part of the noise space. This is $R_{0}<n \leq M$ for the excitation side and $R_{0}<n \leq N$ for the sensor side.

If, as described above, the absolute value of $\boldsymbol{g}(z)$ fluctuates significantly for different $z$, then also the contribution of the error to the image $g(z)^{H} \boldsymbol{\epsilon}$ is significantly different for each $z$.

In order to keep the contribution of the error as constant as possible, it is beneficial to normalise $\boldsymbol{g}(z)$ at every single image point, so that $\|\boldsymbol{g}(z)\|=1$ holds for all $z$, where $\|\bullet\|$ denotes the 2-norm of a vector.

Due to this normalisation, the fluctuation of $\boldsymbol{g}(z)^{H} \boldsymbol{\epsilon}$ is minimized for a given error $\boldsymbol{\epsilon}$. In this case, the influence of the error on the image comes closer to adding a constant to the pseudospectrum, which is proposed in [19] in order to avoid singularities. Adding a constant greater than 0 to the image function does reduce the contrast between $I\left(z \in D_{0}\right)$ and $I\left(z \notin D_{0}\right)$, but does not change the location of the maximum of the image function, as:

$$
\left(\mid \boldsymbol{g}\left(z \in D_{0}\right)^{H} \boldsymbol{\Phi}_{n}+\text { const } \mid\right)^{-1}>\left(\mid \boldsymbol{g}\left(z \notin D_{0}\right)^{H} \boldsymbol{\Phi}_{n}+\text { const } \mid\right)^{-1}
$$

However, as the elements of $\epsilon$ are generally different from each other, the influence of $\boldsymbol{\epsilon}$ on $\boldsymbol{g}_{\Psi, x}^{H} \boldsymbol{\epsilon}$ will be different for each image point. In addition to that, due to $\boldsymbol{\epsilon} \in \mathbb{C}$, it cannot be guaranteed that $\boldsymbol{g}_{\Psi, x}^{H} \boldsymbol{\epsilon}$ is real and greater than 0 . Therefore the error will always add a random contribution to $I(z)$. Nevertheless, the normalisation guarantees that the influence of the error on each image point becomes more comparable. 


\section{References}

[1] C. R. Farrar, K. Worden, Structural Health Monitoring: A Machine Learning Perspective, Wiley, 2013.

${ }_{780}^{4}$ [2] B. W. Drinkwater, P. D. Wilcox, Ultrasonic arrays for non-destructive evaluation: A review, Ndt \& E International 39 (7) (2006) 525-541.

a URL https://ac.els-cdn.com/S0963869506000272/

a 1-s2.0-S0963869506000272-main.pdf?_tid=

b09b6a45-7fb5-46da-891f-5b261e0f8222\&acdnat $=1533646301$

785 f459b1440640d5ee246aa8fd79b174df

[3] S. Chatillon, G. Cattiaux, M. Serre, O. Roy, Ultrasonic non-destructive testing of pieces of complex geometry with a flexible phased array transducer. Ultrasonics 38 (1-8) (2000) 131-134.

q [4] R. Carandente, J. Ma, P. Cawley, The scattering of the fundamental torsional mode from axi-symmetric defects with varying depth profile in pipes, The Journal of 795 the Acoustical Society of America 127 (6) (2010) 3440-3448.

a URL http://scitation.aip.org/content/asa/journal/jasa/127/6/ 10.1121/1.3373406

口 [5] Z. Su, L. Ye, Y. Lu, Guided lamb waves for identification of damage in composite structures: A review, Journal of sound and vibration 295 (3-5) (2006) 753-780.

口[6] D. Alleyne, P. Cawley, The long range detection of corrosion in pipes using lamb waves, in: Review of progress in quantitative nondestructive evaluation, Vol. 14B, 
Springer, 1995, Ch. Chapter 7: Materials' Degradation and Specific Applications, pp. 2073-2080. doi : 10.1007/978-1-4615-1987-4_265.

URL http://lib.dr.iastate.edu/qnde/1995/allcontent/265/

口 [7] M. Kharrat, O. Bareille, W. Zhou, M. Ichchou, Nondestructive assessment of

[9] P. Khalili, P. Cawley, Excitation of single mode lamb waves at high frequency

[10] D. Algernon, H. Ernst, K. Dressler, Signal processing for air-coupled impact-echo plastic elbows using torsional waves: Numerical and experimental investigations, Journal of Nondestructive Evaluation 35 (1) (2016) 1-14.

URL https://link.springer.com/article/10.1007/ s10921-015-0324-6

[8] C.-Y. Kim, K.-J. Park, Mode separation and characterization of torsional guided wave signals reflected from defects using chirplet transform NDT \& E International 74 (2015) 15-23. doi:doi:10.1016/j.ndteint.2015.04.006. URL http://wwW.sciencedirect.com/science/article/pii/ S0963869515000432

thickness products. IEEE transactions on ultrasonics, ferroelectrics, and frequency control 63 (2) (2015) 303-312. doi : 10.1109/TUFFC.2015.2507443

URL http://ieeexplore.ieee.org/stamp/stamp.jsp?tp=\&arnumber= 7352350

using microphone arrays, in: 18th World Conference on Nondestructive Testing, Durban, 2012.

a URL https://wwW.svti.ch/fileadmin/SVTI/NUK/Publikationen/ Signal_Processing.pdf

[11] I. Hertlin, Acoustic resonance analysis for safety parts in mass production, in: Proceedings of 16th World Conference on NDT in Montreal (ndt.net), 2004.

a URL https://wwW.ndt.net/article/wcndt2004/pdf/in-process_ ndt-nde/209_hertlin.pdf

[12] J. Enkvist, O. Svenson, A. Edland, Effects of time pressure and noise on non-destructive testing, Tech. rep., Swedish Nuclear Power Inspectorate (2001). 
[13] I. Hertlin, K. Heldmann, Acoustic resonance analysis in manufacturing-processes and practical applications, Proceedings DGZfP Jahrestagung.

[14] A. Rytter, Vibrational based inspection of civil engineering structures, Ph.D. thesis, Dept. of Building Technology and Structural Engineering, Aalborg University (1993).

口 URL http://vbn.aau.dk/ws/files/18588667/Vibrational_Based_ Inspection_of_Civil_Engineering_Structures

[15] P. Becht, E. Deckers, C. Claeys, B. Pluymers, W. Desmet, Music algorithm for vibro-acoustic defect detection, in: 2nd Euro-Mediterranean Conference on Structural Dynamics and Vibroacoustics - MEDYNA, 2017.

[16] P. Becht, S. van Ophem, E. Deckers, C. Claeys, B. Pluymers, W. Desmet, Model based vibro-acoustic defect detection, KU Leuven - Faculty of Engineering, 2018, Ch. 1, pp. 1-26.

${ }_{850}^{85}$ [17] C. Oestges, A. D. Kim, G. Papanicolaou, A. J. Paulraj, Characterization of space-time focusing in time-reversed random fields, IEEE transactions on antennas and propagation 53 (1) (2005) 283-293.

URL

https://ieeexplore.ieee.org/document/1377603/ \#full-text-section

[18] P. Roux, M. Fink, Time reversal in a waveguide: Study of the temporal and spatial focusing, The Journal of the Acoustical Society of America 107 (5) (2000) 24182429.

URL https://asa.scitation.org/doi/pdf/10.1121/1.428628

[19] S. K. Lehman, A. J. Devaney, Transmission mode time-reversal super-resolution imaging, The Journal of the Acoustical Society of America 113 (5) (2003) 27422753. 
[20] E. A. Marengo, F. K. Gruber, F. Simonetti, Time-reversal music imaging of

URL http://scitation.aip.org/content/asa/journal/jasa/113/5/ $10.1121 / 1.1566975$ extended targets IEEE Transactions on image processing 16 (8) (2007) 19671984.

URL https://ieeexplore . ieee.org/abstract/document/4271531/

[21] H. Lev-Ari, A. Devancy, The time-reversal technique re-interpreted: Subspacebased signal processing for multi-static target location, in: Sensor Array and Multichannel Signal Processing Workshop. 2000. Proceedings of the 2000 IEEE, IEEE, 2000, pp. 509-513.

口 URL https://ieeexplore.ieee.org/stamp/stamp.jsp?tp=\&arnumber= 878061

[22] A. J. Devaney, Time reversal imaging of obscured targets from multistatic data, IEEE Transactions on Antennas and Propagation 53 (5) (2005) 1600-1610. URL http: //ieeexplore.ieee.org/abstract/document/1427917/

[23] A. Baussard, T. Boutin, Time-reversal rap-music imaging, Waves in Random and Complex Media 18 (1) (2008) 151-160. URL http://wWW.tandfonline.com/doi/pdf/10.1080/ $17455030701481856 ?$ needAccess $=$ true

[24] M. E. Yavuz, F. L. Teixeira, On the sensitivity of time-reversal imaging techniques to model perturbations. IEEE transactions on antennas and propagation 56 (3) (2008) 834-843.

URL http://ieeexplore.ieee.org/abstract/document/4463920/

885 [25] W. Zhang, A. Hoorfar, L. Li, Through-the-wall target localization with time reversal music method, Progress In Electromagnetics Research 106 (2010) 75-89. URL http://jpier .org/PIER/pier.php?paper $=10052408$

[26] Y. OGAWA, T. NISHIMURA, T. OHGANE, Time-reversal music imaging with time-domain gating technique, IEICE transactions on communications 95 (7) 
(2012) 2377-2385.

口

URL http://eprints.lib.hokudai.ac.jp/dspace/bitstream/2115/ 49742/1/ToCE95B-7_2377-2385.pdf

[27] X.-F. Liu, B.-Z. Wang, J. L.-W. Li, Transmitting-mode time reversal imaging using music algorithm for surveillance in wireless sensor network, IEEE Transactions on Antennas and Propagation 60 (1) (2012) 220-230.

a URL http://ieeexplore.ieee.org/stamp/stamp.jsp?arnumber= 6018267

[28] R. Solimene, A. Dell'Aversano, G. Leone, Interferometric time reversal music for small scatterer localization, Progress In Electromagnetics Research 131 (2012) 243-258.

URL http://mobile.jpier.org/PIER/pier131/15.12062103.pdf

[29] J. He, F.-G. Yuan, Lamb wave-based subwavelength damage imaging using the dort-music technique in metallic plates, Structural Health Monitoring 15 (1) (2016) 65-80. doi : 10.1177/1475921715623359

URL http://shm.sagepub.com/content/early/2016/01/06/ 1475921715623359.abstract

[30] M. E. Yavuz, F. L. Teixeira, Full time-domain dort for ultrawideband electromagnetic fields in dispersive, random inhomogeneous media, IEEE Transactions on Antennas and Propagation 54 (8) (2006) 2305-2315.

910 प URL http://ieeexplore.iee.org/stamp/stamp.jsp?tp=\&arnumber= 1668305

[31] C. Fan, M. Pan, F. Luo, B. W. Drinkwater, Multi-frequency time-reversal-based imaging for ultrasonic nondestructive evaluation using full matrix capture, IEEE transactions on ultrasonics, ferroelectrics, and frequency control 61 (12) (2014) 2067-2074.

URL https://ieeexplore. ieee.org/abstract/document/6968700

[32] F. Deng, F. L. Teixeira, Locating small structural damages in pipes using space-frequency dort processing, Results in Physics 7 (2017) 1637-1643. 
[33] A. E. Fouda, F. L. Teixeira, Statistical stability of ultrawideband time-reversal imaging in random media, IEEE Transactions on Geoscience and Remote Sensing 52 (2) (2014) 870-879.

URL http://ieeexplore.ieee.org/abstract/document/6481435/

[34] C. Prada, M. Fink, Eigenmodes of the time reversal operator: A solution to selective focusing in multiple-target media. Wave Motion 20 (2) (1994) 151-163. URL http://wWw.sciencedirect.com/science/article/pii/ 0165212594900396

[35] C. Prada, S. Manneville, D. Spoliansky, M. Fink, Decomposition of the time reversal operator: Detection and selective focusing on two scatterers, The Journal of the Acoustical Society of America 99 (4) (1996) 2067-2076.

口

URL http://scitation.aip.org/content/asa/journal/jasa/99/4/ 10.1121/1.415393

[36] R. Solimene, G. Ruvio, A. Dell'Aversano, A. Cuccaro, M. J. Ammann, R. Pierri, Detecting point-like sources of unknown frequency spectra, Progress In Electromagnetics Research B 50 (2013) 347-364.

URL http://jpier .org/PIERB/pier .php?paper=13030414

[37] R. J. Allemang, D. L. Brown, A correlation coefficient for modal vector analysis, in: Proceedings of the 1st international modal analysis conference, Vol. 1, SEM Orlando, 1982, pp. 110-116.

1. URL http://wWW.sdrl.uc.edu/sdrl/referenceinfo/documents/ papers/imac1982-mac.pdf 